\title{
Induction of peroxisomal lipid metabolism in mice fed a high-fat diet
}

\author{
SACHI KOZAWA ${ }^{1,4}$, AYAKO HONDA ${ }^{1}$, NAOMI KAJIWARA ${ }^{1}$, YASUHIKO TAKEMOTO ${ }^{1}$, TOMOKO NAGASE ${ }^{1}$, \\ HIDEKI NIKAMI $^{2}$, YUKIO OKANO ${ }^{3}$, SHIGERU NAKASHIMA ${ }^{4}$ and NOBUYUKI SHIMOZAWA ${ }^{1}$ \\ Divisions of ${ }^{1}$ Genomic Research, and ${ }^{2}$ Animal Experiment, Life Science Research Center, Gifu University; \\ ${ }^{3}$ Gifu University (trustee); ${ }^{4}$ Department of Cell Signaling, Division of Molecular and Cellular Biology, \\ Gifu University Graduate School of Medicine, Gifu 501-1193, Japan
}

Received April 1, 2011; Accepted August 8, 2011

DOI: $10.3892 / \mathrm{mmr} .2011 .560$

\begin{abstract}
Peroxisomes catalyze a range of essential metabolic functions, mainly related to lipid metabolism. However, their roles in obesity have yet to be clarified. The aim of this study was to investigate the correlation between obesity and peroxisomal lipid metabolism, particularly very long-chain fatty acid (VLCFA) metabolism, gene expression of peroxisomal $\beta$-oxidation enzymes, peroxisomal ATP-binding cassette (ABC) transporter adrenoleukodystrophy (ABCD1) gene and its related gene, ABCD2, the elongation of the VLCFA (ELOVL) gene family and the transcriptional factors involved in the regulation of these genes, including peroxisome proliferator-activated receptor $\alpha$ (PPAR $\alpha)$ and sterol regulatory element-binding protein. These factors were analyzed in livers from mice fed a high-fat diet (HFD) or a regular diet (RD) for 20 weeks. Furthermore, the amounts of plasma saturated and unsaturated fatty acids, including VLCFAs, were measured. A HFD induced hepatic gene expression of not only hydroxysteroid 17- $\beta$ dehydrogenase 4 (HSD17b4) and sterol carrier protein 2 (SCP2) in peroxisomal $\beta$-oxidation enzymes but also of ELOVL1, 2, 5 and 6, which are involved in the elongation of saturated and unsaturated VLCFAs. Furthermore, ABCD2 mRNA prominently increased in the HFD mice. The transcriptional regulator of these genes, PPAR $\alpha$, was also up-regulated in the HFD mice. VLCFA ratios including C24:0/ $\mathrm{C} 22: 0, \mathrm{C} 25: 0 / \mathrm{C} 22: 0$ and $\mathrm{C} 26: 0 / \mathrm{C} 22: 0$ are the most significant diagnostic markers of inherited peroxisomal diseases. These ratios were found to be low in the plasma of the HFD mice compared with the RD mice. The results suggest that HFD activates hepatic peroxisomal VLCFA metabolism, and may provide useful fundamental information to explain the role of peroxisomal function in obesity and lifestyle-related diseases.
\end{abstract}

Correspondence to: Dr Nobuyuki Shimozawa, Division of Genomic Research, Life Science Research Center, Gifu University, 1-1 Yanagido, Gifu 501-1193, Japan

E-mail: nshim@gifu-u.ac.jp

Key words: obesity, peroxisomal beta oxidation, very long-chain fatty acids

\section{Introduction}

Peroxisomes are single-membrane-lined organelles present in all eukaryotic cells, which catalyze a range of essential metabolic functions including $\beta$-oxidation of VLCFA and the synthesis of bile acids and plasmalogen in mammals. Inborn errors of peroxisomal metabolism in humans, causing peroxisomal diseases, consist mainly of two large groups. The first group comprises peroxisomal biogenesis disorders (PBDs), which are characterized by fetal autosomal recessive diseases with no effective therapy. These disorders are caused by a defect in PEX genes, which encode peroxins, proteins necessary for the biogenesis of peroxisomes and the import of the peroxisomal matrix and membrane proteins, resulting in the generalized loss of metabolic function of peroxisomes. The second group includes diseases with single enzyme deficiencies, including peroxisomal $\beta$-oxidation such as straight-chain acyl CoA oxidase (ACOX1) and D-bifunctional protein (DBP). X-linked adrenoleukodystrophy (ALD) is the most common peroxisomal disease, with impaired $\beta$-oxidation of saturated VLCFA resulting in the accumulation of VLCFA in tissues and plasma. ALD is caused by a defect of the ABCD1 gene, which encodes the peroxisomal ABC transporter ALD protein. Since most of these peroxisomal diseases with $\beta$-oxidation dysfunction reveal the accumulation of VLCFA, increased VLCFA in the plasma has been used as a diagnostic marker for these diseases (1).

The genes encoding the peroxisomal $\beta$-oxidation pathway in the liver are transcriptionally regulated by PPAR $\alpha$, which is a nuclear hormone receptor (2). PPAR $\alpha$ plays a key role in energy expenditure, and its substrates involved in fatty acid oxidation containing VLCFA may function as PPAR $\alpha$ ligands (3). On the other hand, it has recently been shown that administration of a HFD in mice resulted in the up-regulation of hepatic PPAR $\alpha$ mRNA (4). These results suggest the possible involvement of peroxisomal metabolism in obesity. Therefore, the regulation of PPAR $\alpha$ activity through modulating endogenous ligands may have the potential to generate a new therapeutic approach for obesity and lifestyle-related diseases (5). In the present study, in order to obtain insight into the role of peroxisomal metabolism, particularly VLCFA metabolism in obesity, we compared the levels of VLCFAs 
in plasma. We also compared the mRNA levels of the genes involved in peroxisomal $\beta$-oxidation, ALD and ALD-related protein (ABCD1 and 2), and elongation of VLCFAs along with their regulator genes, PPAR $\alpha$, retinoid $\mathrm{X}$ receptor $\alpha(\mathrm{RXR} \alpha)$ and sterol regulatory element-binding protein (SREBP), in the livers of HFD-feeding and regular diet (RD)-feeding mice.

\section{Materials and methods}

Mice and diets. C57BL/6J Jcl female mice in their 14th day of pregnancy were obtained from Clea Japan Inc. (Tokyo, Japan) and housed in two groups: one group was fed on a HFD (HFD32, Clea Japan Inc.) and the other was fed on a RD (CE2, Clea Japan Inc.). The mice were allowed access to water and a HFD or a RD (for diet composition see Table I). The regimens were maintained following delivery of the pups. Following weaning (at day 21), females were excluded from further study, and we analyzed only male pups in the two groups fed on RD and HFD. The body weight of mice in this study was measured every 2 or 3 weeks. The animals were maintained under controlled light (12-h light/dark cycle, light on at 08:00) and temperature $\left(23 \pm 2^{\circ} \mathrm{C}\right)$ conditions. All of the animal care and experimental procedures were conducted under the regulations for animal experiments of Gifu University.

Blood collection for biochemical analysis. Blood samples $(0.3 \mathrm{ml})$ were drawn at week 6 and 20 from the submandibular veins of the mice with a goldenrod animal lancet (MEDI Point, NY, USA) in a $1.5 \mathrm{ml}$ tube with $5 \mu \mathrm{l}$ coagulant (Wako, Japan). Ethics and experimental procedures were approved by the Committee for Animal Research and Welfare of Gifu University. Samples were centrifuged at 2,500 x g for 15 min at room temperature and plasma was collected and stored at $-20^{\circ} \mathrm{C}$ until analysis. Leptin and adiponectin levels in plasma from mice were measured by Oriental Yeast Co., Ltd. at week 20 using commercial kits for ELISA (Morinaga Institute of Biological Science Inc. and Otsuka Pharmaceutical Co., Ltd, respectively). Measurements of saturated and unsaturated fatty acids were taken, including ratios of C24:0/C22:0, C25:0/C22:0, C26:0/C22:0 and C16:0 DMA (plasmalogen)/C16:0 in $50 \mu \mathrm{l}$ plasma from mice, using gas chromatography/mass spectrometry (GC/MS) as previously described (6).

Tissue collection and real-time quantitative PCR. Mice were sacrificed by cervical dislocation at week 20 , and the livers were removed and preserved in RNAlater (Applied Biosystems, Tokyo, Japan), and stored at $-20^{\circ} \mathrm{C}$ for RNA preparation. Total RNA was purified with Isogen (Nippon Gene, Japan) and cDNA was synthesized with M-MLV reverse transcriptase (Invitrogen, Japan). Real-time PCR analysis was carried out in an ABI PRISM 7000 using fluorescent TaqMan methodology. Real-time quantitative PCR was performed for each of the following genes, using ready-to use primer and probe sets pre-developed by Applied Biosystems (TaqMan gene expression assays): ACOX1 (Mm00443579_m1), ACOX2 (Mm00446408_m1), HSD17b4 (Mm00500443_m1), acetyl-Coenzyme A acyltransferase 1A (ACAA1, Mm00728460_s1), SCP2 (Mm01257982_m1), ABCD1 (Mm00431749_m1), ABCD2
Table I. Fatty acids composition of experimental diets

\begin{tabular}{lcc}
\hline & $\begin{array}{c}\text { Regular diet } \\
\text { (CE2) }\end{array}$ & $\begin{array}{c}\text { High-fat diet } \\
\text { (HFD32) }\end{array}$ \\
\cline { 2 - 3 } Crude lipid content & $4.63 / 100 \mathrm{~g}$ & $\begin{array}{c}31.9 / 100 \mathrm{~g} \\
100 \%\end{array}$ \\
\hline Composition of fatty acids & $100 \%$ & 1.1 \\
C14:0 & 0.82 & 0.3 \\
C15:1 & $\mathrm{a}$ & 0.1 \\
C16:0 & $\mathrm{a}$ & 12.6 \\
C16:1 & 16.25 & 1.2 \\
C17:0 & 1.34 & 0.4 \\
C17:1 & 0.52 & 0.3 \\
C18:0 & $\mathrm{a}$ & 7.5 \\
C18:1 & 2.13 & 64.3 \\
C18:2 & 23.06 & 10.2 \\
C18:3 & 44.44 & 0.2 \\
C20:0 & 3.26 & 0.3 \\
C20:1 & 0.49 & 0.3 \\
C20:4 & 0.72 & $\mathrm{a}$ \\
C20:5 & $\mathrm{a}$ & $\mathrm{a}$ \\
C22:0 & 2.27 & 0.3 \\
C22:6 & 0.25 & $\mathrm{a}$ \\
C24:0 & 1.29 & $\mathrm{a}$ \\
C24:1 & 0.18 & 0.8 \\
Unidentified & 0.20 & \\
\hline
\end{tabular}

${ }^{\text {a }}$ Included in the unidentified composition.

(Mm00496455_m1), PPAR $\alpha$ (Mm00627559_m1), SREBP-1c (Mm01138344_m1), Elovl1 (Mm00517077_m1), Elovl2 (Mm00517086_m1), Elovl3 (Mm00468164_m1), Elovl4 (Mm00521704_m1), Elovl5 (Mm00506717_m1), Elovl6 (Mm00851223_s1), Elovl7 (Mm00512434_m1), leptin receptor (LEPR, Mm00440181_m1), tumor necrosis factor (TNF, Mm00443258_m1), adiponectin (Adipoq, Mm00456425_m1), adiponectin receptor 1 (Adipor1, Mm01291334_mH), Adipor2 (Mm01184031_g1), monocyte chemoattractant protein 1 (MCP1, Mm00441242_m1), uncoupling protein 2 (UCP2, Mm00627599_m1), fatty acid synthase (FASN, Mm00662319_m1), RXR $\alpha$ (Mm01332431_m1), thyroid hormone receptor $\beta$ (THR $\beta$, Mm00437044_m1) and eukaryotic 18S rRNA (18S, Hs99999901_s1) as an endogenous control. The mRNA Ct values for these genes were normalized to eukaryotic 18S rRNA and expressed as a relative increase or decrease in the liver from the HFD mice to those in the RD mice.

Statistical analysis. Results are expressed as the means \pm standard deviation (SD). Statistical analysis was performed using the F-test and two-tailed t-test for RT-PCR and the F-test and one-tailed t-test for other experiments. 

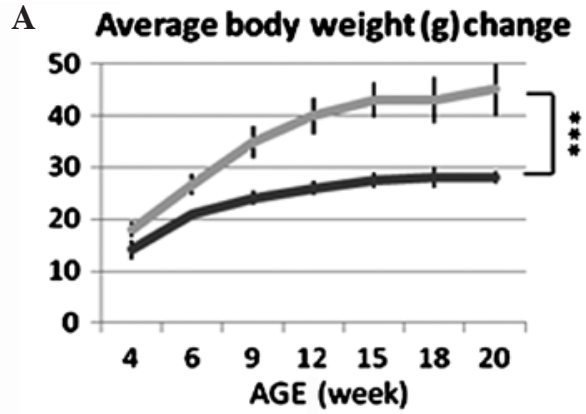

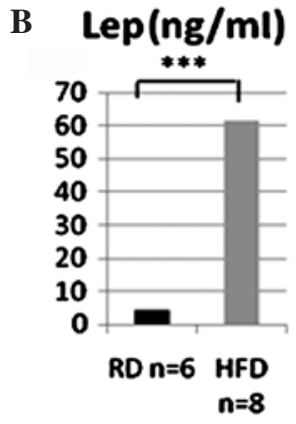

\section{C $A D N(\mathrm{mg} / \mathrm{ml})$}

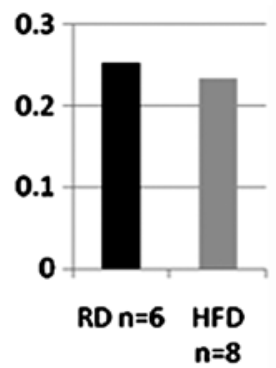

RD Average $n=7 \longrightarrow$ HFD Average $n=8$

Figure 1. Body weight change, plasma leptin and adiponectin levels in regular and HFD mice. (A) The means \pm SD of body weight change. (B) Plasma leptin level (Lep) (ng/ml). (C) Plasma adiponectin level (ADN) (mg/ml). Statistically significant differences by the Student's t-test are shown by ${ }^{* * *}$ p $<0.005$ RD vs. HFD.

A

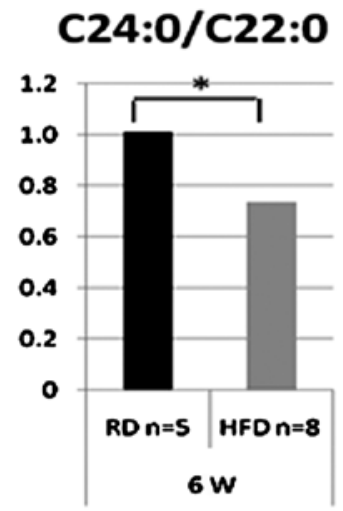

$\mathbf{E}$

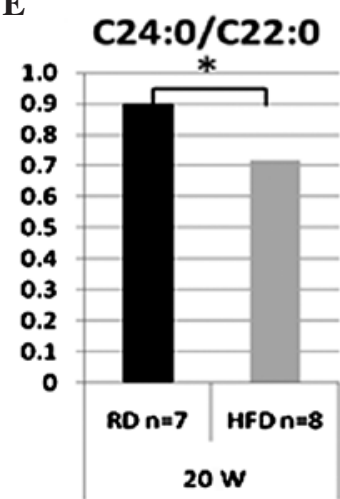

B

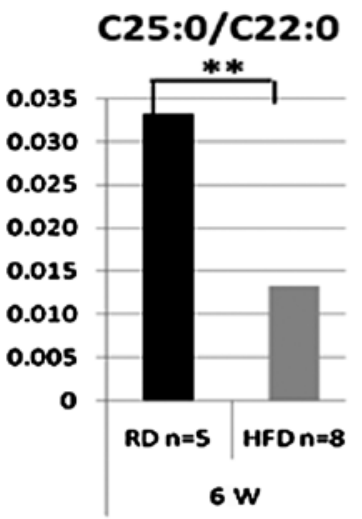

$\mathbf{F}$

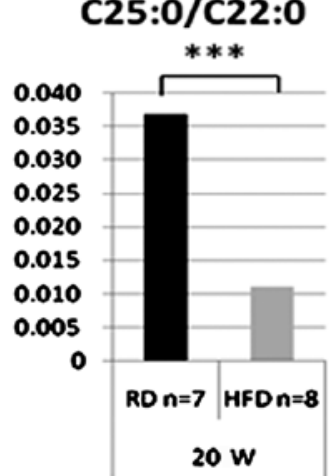

C

C26:0/C22:0

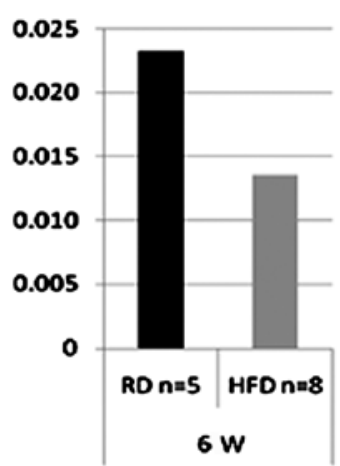

G

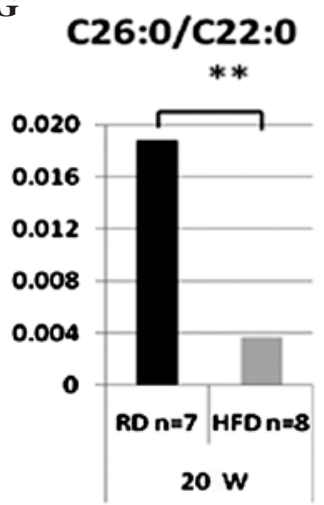

D

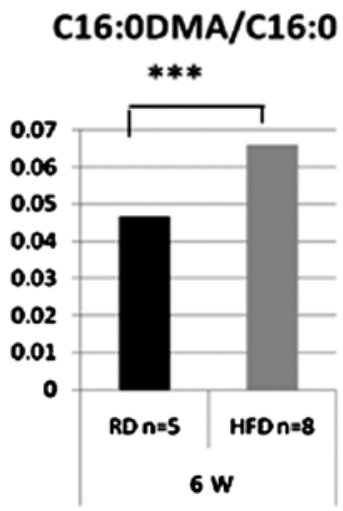

H

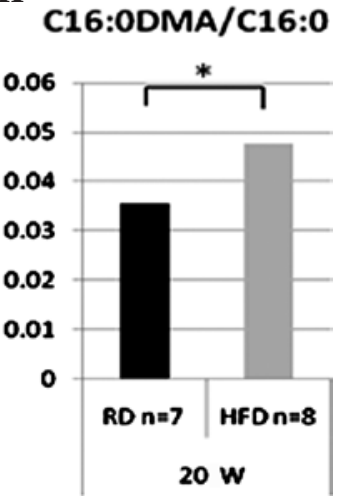

Figure 2. Plasma VLCFA levels in the RD and HFD mice. The ratios of (A and E) C24:0/C22:0, (B and F) C25:0/C22:0, (C and G) C26:0/C22:0 and (D and H) C16:0 DMA/C16:0 in plasma from RD and HFD mice at weeks 6 and 20, respectively. Statistically significant differences using the Student's t-test are shown by ${ }^{*} \mathrm{p}<0.05,{ }^{* *} \mathrm{p}<0.01,{ }^{* * *} \mathrm{p}<0.005, \mathrm{RD}$ vs. HFD, respectively (w, weeks).

\section{Results}

Body weight gain, plasma leptin and adiponectin levels. Growth curves of mice fed with $\operatorname{HFD}(n=8)$ or RD $(n=7)$ are shown in Fig. 1A. At week 20, body weight of the HFD mice was prominently increased compared to that of the RD mice (RD, 28.08 $\pm 1,153 \mathrm{~g}$; HFD, 45.03 $\pm 5,081 \mathrm{~g}, \mathrm{p}<0.005$ ). The plasma leptin level of the HFD mice was also markedly increased compared to that of the RD mice (Fig. 1B), whereas no difference was observed in plasma adiponectin levels between HFD and RD (Fig. 1C).
VLCFA. We compared the ratios of C24:0/C22:0, C25:0/C22:0 and $\mathrm{C} 26: 0 / \mathrm{C} 22: 0$ in plasma between the HFD and RD mice at weeks 6 and $20(\mathrm{HFD}, \mathrm{n}=8 ; \mathrm{RD}, \mathrm{n}=5$ and $\mathrm{n}=7$, at weeks 6 and 20, respectively). $\mathrm{C} 24: 0 / \mathrm{C} 22: 0$ and $\mathrm{C} 25: 0 / \mathrm{C} 22: 0$ ratios in the HFD mice at week 6 were significantly lower than those in RD mice (Fig. 2A and B), and three VLCFA ratios in the HFD mice at week 20 were significantly lower compared to those in the RD mice (Fig. 2E-G). By contrast, plasmalogen content (C16:0 DMA/C16:0) in the HFD mice was higher than that of the RD mice at both 6 and 20 weeks (Fig. 2D and H). These results suggest that peroxisomal lipid metabolism, including 
Table II. Saturated and unsaturated fatty acids in plasma from feeding HFD and RD mice at 20 weeks of age.

\begin{tabular}{lcc}
\hline & RD (average) $\mathrm{n}=7$ & HFD (average) $\mathrm{n}=8$ \\
\cline { 2 - 3 } & $\mu \mathrm{g} / 50 \mu 1$ plasma & $\mu \mathrm{g} / 50 \mu 1$ plasma \\
\hline C14:0 & $0.853^{\mathrm{a}}$ & $0.551^{\mathrm{a}}$ \\
C14:1 & 0.037 & 0.037 \\
C16:0 DMA & $1.297^{\mathrm{a}}$ & $1.682^{\mathrm{a}}$ \\
C16:0 & 35.833 & 36.873 \\
C16:1 & $6.233^{\mathrm{a}}$ & $2.723^{\mathrm{a}}$ \\
$\mathrm{C} 18: 0$ & $12.257^{\mathrm{a}}$ & $24.188^{\mathrm{a}}$ \\
C18:1 & $30.413^{\mathrm{a}}$ & $49.772^{\mathrm{a}}$ \\
$\mathrm{C} 18: 2$ & $52.831^{\mathrm{b}}$ & $42.555^{\mathrm{b}}$ \\
C18:3 & $1.763^{\mathrm{a}}$ & $0.717^{\mathrm{a}}$ \\
C20:0 & 0.358 & 0.282 \\
C20:1 & 1.400 & 1.032 \\
ARA & $5.107^{\mathrm{a}}$ & $21.174^{\mathrm{a}}$ \\
EPA & $5.445^{\mathrm{a}}$ & $1.425^{\mathrm{a}}$ \\
C22:0 & $0.372^{\mathrm{b}}$ & $0.508^{\mathrm{b}}$ \\
C22:1 & 0.155 & 0.141 \\
C24:0 & 0.312 & 0.365 \\
C24:1 & $1.192^{\mathrm{a}}$ & $2.212^{\mathrm{a}}$ \\
DHA & 16.265 & 20.148 \\
C25:0 & $0.017^{\mathrm{a}}$ & $0.006^{\mathrm{a}}$ \\
C26:0 & $0.0086^{\mathrm{b}}$ & $0.002^{\mathrm{b}}$ \\
\hline
\end{tabular}

Statistically significant differences by the Student's t-test are shown by ${ }^{\mathrm{a}} \mathrm{p}<0.005,{ }^{\mathrm{b}} \mathrm{p}<0.05$, respectively. C16:0 DMA, plasmalogen; ARA, arachidonic acid; EPA, eicosapentanoic acid; DHA, docosahexaenoic acid.

$\beta$-oxidation of VLCFA and plasmalogen biosynthesis, were activated in the HFD mice. Further results including levels of saturated and unsaturated fatty acids in the HFD and RD mice at week 20 are shown in Table II.

Quantitative real-time $P C R$. Concerning peroxisomal $\beta$-oxidation enzymes, the hepatic gene expression of HSD17 $\beta 4$ and SCP2 was significantly up-regulated in the HFD mice at week 20, as compared to the RD mice. Furthermore, peroxisomal ABCD2 mRNA was markedly higher in the HFD mice (Fig. 3A). Among the ELOVL gene family, ELOVL1, 2, 5 and 6 were up-regulated in the HFD mice (Fig. 3B). The level of ELOVL7 mRNA was elevated 12-fold in the HFD mice, although the change was not statistically significant. Additionally, among the regulatory genes of these enzyme genes, PPAR $\alpha$ increased significantly in the HFD mice. The SREBF regulating gene involved in fatty acid synthesis revealed a tendency to increase in the HFD mice, but this was not statistically significant. Further real-time PCR analysis of hepatic gene expression revealed that mRNA levels of TNF, Adipor2, MCP1, UCP2 and FASN were elevated, and that of LEPR was significantly down-regulated in the HFD mice, as compared to the RD mice (Fig. 3C).

\section{Discussion}

Peroxisomes are involved in a number of essential functions in mammals, mainly related to lipid metabolism, including fatty acid $\beta$-oxidation, plasmalogen biosynthesis and phytanic acid $\alpha$-oxidation. The significance of peroxisomes for humans is stressed by the existence of an expanding group of human diseases in which peroxisome function is impaired (7). The peroxisomal $\beta$-oxidation metabolizes substrates including VLCFAs such as C24:0 and C26:0, pristanic acid and the bile acid intermediates. Inborn errors of peroxisomal metabolism are mainly divided into PBDs and single peroxisomal enzyme deficiencies (1). Most of these peroxisomal diseases involve $\beta$-oxidation dysfunction. Therefore, the accumulation of VLCFAs in the plasma has been used as a diagnostic marker for these diseases (1). Furthermore, we have established a diagnostic system for peroxisomal diseases in Japan, using measurements of increased VLCFA and phytanic acid, and decreased plasmalogen in plasma from the patients (6).

A nuclear transcription factor, PPAR $\alpha$, forms a heterodimer with the RXR and binds to target genes involved in peroxisome proliferation and fatty acid metabolism, widely including $\beta$-oxidation. HFD is the major cause of obesity and in wildtype HFD mice it was associated with a significant increase in hepatic PPAR $\alpha$ gene expression. Activation of PPAR $\alpha$ leads to an increase in tissue-specific expression of the key genes involved in fatty acid uptake and $\beta$-oxidation $(4,8)$.

To elucidate the role of peroxisomal lipid metabolism in obesity, we compared HFD and RD mice. The dams in the HFD group were administered HFD at day 14 of their pregnancy. The HFD mice gained body weight increasingly as compared with the RD mice (Fig. 1A). The plasma leptin level in the HFD mice was significantly higher (approximately 14-fold) than that in RD mice, whereas no difference in adiponectin levels was observed between the HFD and RD mice (Fig. 1B and C).

Of note, HFD induced a decrease in VLCFA levels (C24:0/ C22:0, C25:0/C22:0 and C26:0/C22:0) and an increase in plasmalogen levels (C16:0DMA/C16:0) in plasma, compared with the RD (Fig. 2), suggesting the possible activation of hepatic peroxisomal function by HFD. Therefore, mRNA expression levels of peroxisomal $\beta$-oxidation enzymes including ACOX1, HSD17 $\beta 4$, AACA1, and their regulatory genes, PPAR $\alpha$, SREBP, MCP1 and UCP2 were analyzed. Most of the hepatic mRNAs involved in peroxisomal $\beta$-oxidation, and also their regulatory genes, were up-regulated in the HFD mice compared with the RD mice (Fig. 3). These results indicate that HFD may induce peroxisomal metabolism in the liver.

Furthermore, the mRNA expression level of ABCD2 increased 6-fold in the HFD mice. ABCD2 is speculated to act as a dimerization partner of $\mathrm{ABCD1}$, the X-linked adrenoleukodystrophy-associated protein (9), and has a functional redundancy with $\mathrm{ABCD} 1$. Therefore, mRNA expression levels of RXR $\alpha$ and THR $\beta$, as well as PPAR $\alpha$ and SREBP, which were reported to be regulators of $\mathrm{ABCD} 2$ expression were assessed (10). Only PPAR $\alpha$ mRNA expression was found to be higher in the HFD mice than in the RD mice (Fig. 3).

The ELOVL gene family comprises of 7 distinct fatty acid elongase subtypes (Elovl1-7). Each elongase has a distinct tissue distribution, and the individual enzymes exhibit 
A

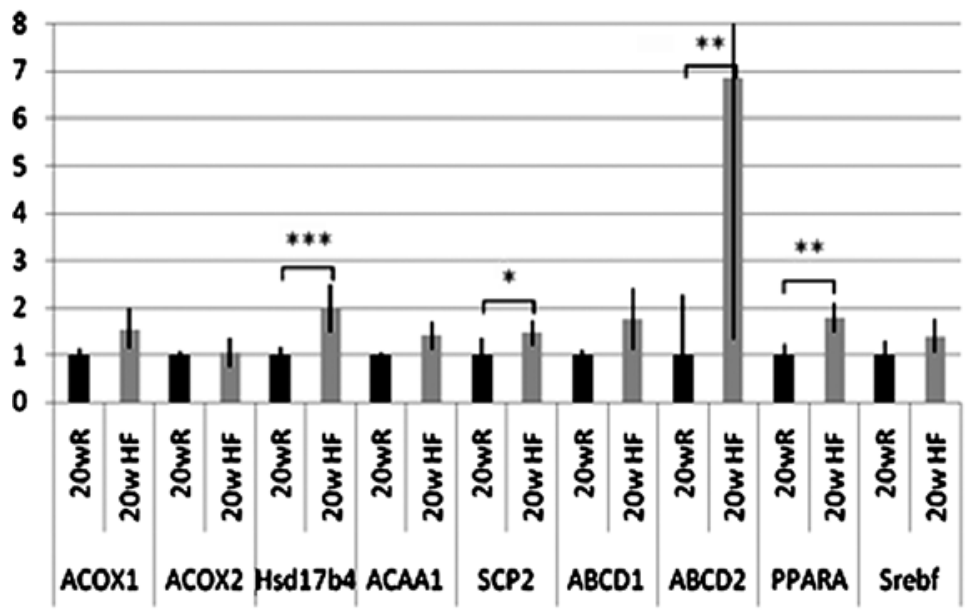

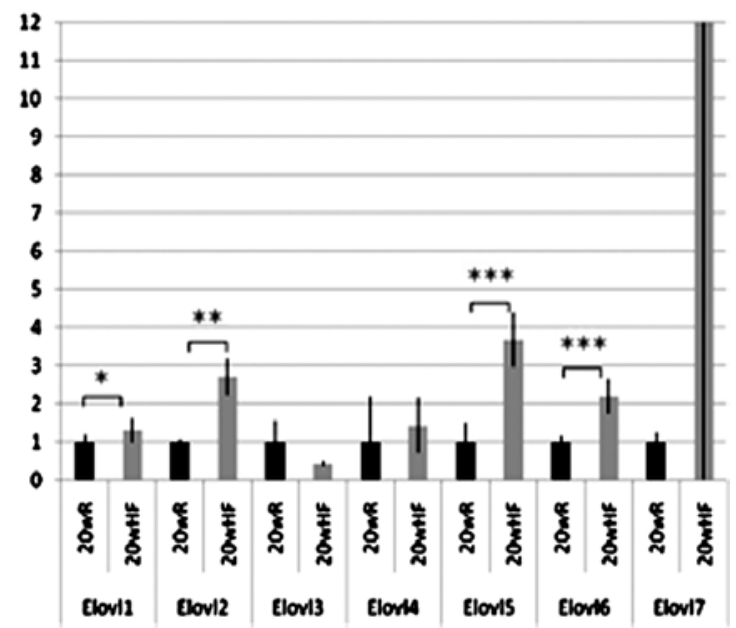

C

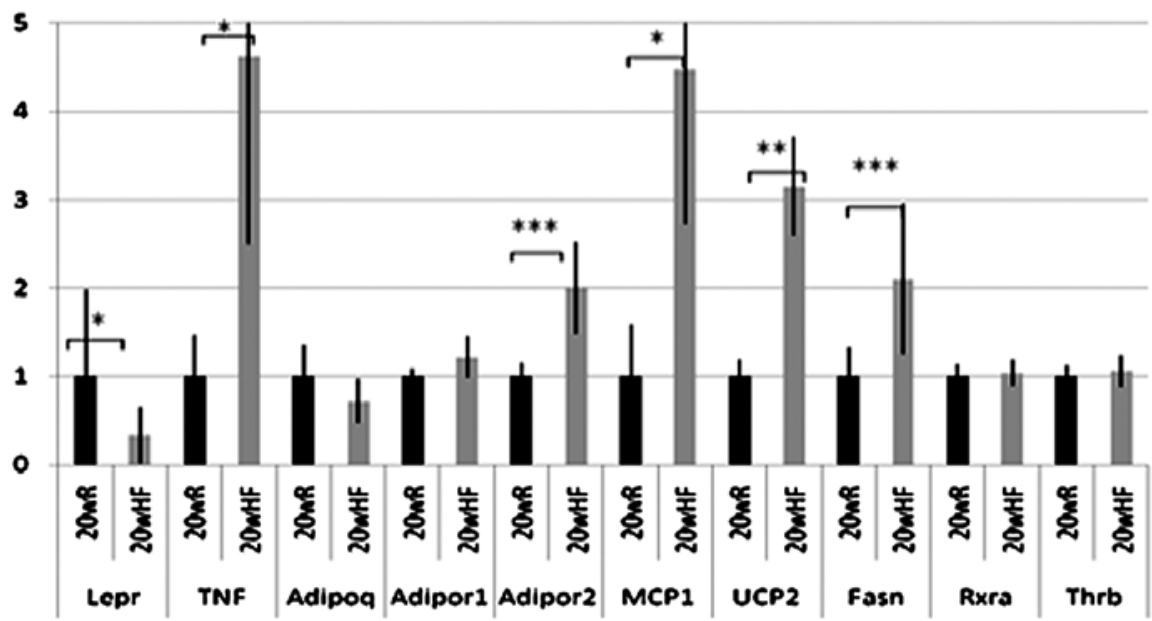

Figure 3. Hepatic gene expression in RD and HFD mice. Data were calculated relative to the expression of 18S rRNA. The F-test and two-tailed t-test are indicated by ${ }^{*} \mathrm{p}<0.05,{ }^{* *} \mathrm{p}<0.01,{ }^{* * *} \mathrm{p}<0.005, \mathrm{RD}$ vs. HFD, respectively (w, weeks).

different fatty acid substrate preferences. The metabolic pathways of long-chain fatty acids play a significant role in the membrane lipid composition as well as in the generation of precursors for cell signaling molecules. In this study, Elovl7 mRNA was up-regulated 12-fold, and levels of Elovl1, 2, 5 and 6 were also higher in the HFD mice (Fig. 3B). Elovl7 expression is regulated by lipogenic enzymes and the androgen pathway through SREBP (11). Only Elovl3 revealed a decreasing tendency in the HFD mice (Fig. 3B). Brolinson et al (12) reported similar results, revealing that Elovl3 expression was found to be suppressed in ABCD2-overexpressing mice.

A HFD is the major cause of obesity, which increases oxidative stress accompanied by an elevation of NADPH oxidase expression. Production of selective reactive oxygen species in the adipose tissue of obese mice leads to the development of inflammatory processes and to the dysregulation of adipocytokines, including adiponectin, plasminogen activator inhibitor-1 and IL-6 (13). Furthermore, a variety of dietary components may play significant roles in the development of insulin resistance (14), which may affect the progression of diabetes (15), hepatic steatosis (16), cardiovascular disease and hypertension (17).

Peroxisomal lipid metabolism has emerged as an essential pathway related to lifestyle-related diseases, including obesity, diabetes and cardiovascular disease. Gaining further insight into the peroxisomal metabolic pathway may provide useful information to develop new therapeutic approaches to these diseases. The data revealing peroxisomal metabolites and gene expression in the HFD mice may provide useful information for the understanding of these lifestyle-related diseases.

\section{Acknowledgements}

The authors thank Dr Y. Okazaki and Dr T. Imanaka for their helpful comments. This study was supported by a grant-in-aid for Scientific Research (21591318) from the Japan Society for the Promotion of Science, a grant for Child Health and Development and a grant for Research on Intractable Diseases from the Health and Labor Sciences Research Grants. 


\section{References}

1. Shimozawa N: Molecular and clinical aspects of peroxisoma diseases. J Inherit Metab Dis 30: 193-197, 2007.

2. Reddy JK and Hashimoto T: Peroxisomal $\beta$-oxidation and peroxisome proliferator-activated receptor $\alpha$ : an adaptive metabolic system. Annu Rev Nutr 21: 193-230, 2001.

3. Hostetler HA, Kier AB and Schroeder F: Very-long-chain and branched-chain fatty acyl-CoAs are high affinity ligands for the peroxisome proliferator-activated receptor $\alpha$ (PPAR $\alpha)$. Biochemistry 45: 7669-7681, 2006.

4. Patsouris D, Reddy JK, Müller M and Kersten S: Peroxisome proliferator-activated receptor $\alpha$ mediates the effects of high-fat diet on hepatic gene expression. Endocrinology 147: 1508-1516, 2006.

5. Pyper SR, Viswakarma N, Jia Y, Zhu YJ, Fondell JD and Reddy JK: PRIC295. A nuclear receptor coactivator, identified from PPAR- $\alpha$ interacting cofactor complex. PPAR Res: Sep 5, 2010 (E-pub ahead of print).

6. Takemoto Y, Suzuki Y, Horibe R, Shimozawa N, Wonders RJ and Kondo N: Gas chromatography/mass spectrometry analysis of very long chain fatty acids, docosahexaenoic acid, phytanic acid and plasmalogen for the screening of peroxisomal disorders. Brain Dev 27: 481-487, 2003.

7. Wanders RJ, Vreken P, Ferdinandusse S, et al: Peroxisomal fatty acid $\alpha$ - and $\beta$-oxidation in humans: enzymology, peroxisomal metabolite transporters and peroxisomal diseases. Biochem Soc Trans 2: 250-267, 2001.

8. Fruchart JC: Peroxisome proliferator-activated receptor- $\alpha$ (PPAR $\alpha$ ): at the crossroads of obesity, diabetes and cardiovascular disease. Atherosclerosis 205: 1-8, 2009.
9. Liu LX, Janvier K, Berteaux-Lecellier V, Cartier N, Benarous R and Aubourg P: Homo- and heterodimerization of peroxisomal ATP-binding cassette half-transporters. J Biol Chem 12: 32738-32743, 1999.

10. Weinhofer I, Kunze M, Rampler H, et al: Distinct modulatory roles for thyroid hormone receptors TR $\alpha$ and TR $\beta$ in SREBP1activated ABCD2 expression. Eur J Cell Biol 87: 933-945, 2008.

11. Tamura K, Makino A, Hullin-Matsuda F, et al: Novel lipogenic enzyme ELOVL7 is involved in prostate cancer growth through saturated long-chain fatty acid metabolism. Cancer Res 69: 8133-8140, 2009.

12. Brolinson A, Fourcade S, Jakobsson A, Pujol A and Jacobsson A: Steroid hormones control circadian Elovl3 expression in mouse liver. Endocrinology 149: 3158-3166, 2008.

13. Furukawa S, Fujita T, Shimabukuro M, et al: Increased oxidative stress in obesity and its impact on metabolic syndrome. J Clin Invest 114: 1752-1761, 2004.

14. Morrison CD, Huypens P, Stewart LK and Gettys TW: Implications of crosstalk between leptin and insulin signaling during the development of diet-induced obesity. Biochim Biophys Acta 1792: 409-416, 2009.

15. Kadowaki $\mathrm{T}$ and Yamauchi T: Adiponectin and adiponectin receptors. Endocr Rev 26: 439-451, 2005.

16. Pessayre D, Fromenty B and Mansouri A: Mitochondrial injury in steatohepatitis. Eur J Gastroenterol Hepatol 16: 1095-1105, 2004.

17. Sharma N, Okere IC, Duda MK, Chess DJ, O'Shea KM and Stanley WC: Potential impact of carbohydrate and fat intake on pathological left ventricular hypertrophy. Cardiovasc Res 73: 257-268, 2007. 